\title{
DERIVATIVES OF ADDITION THEOREMS FOR LEGENDRE FUNCTIONS
}

\author{
D.E. WINCH ${ }^{1}$ and P.H. ROBERTS ${ }^{2}$
}

(Received 1 March 1994; revised 28 May 1994)

\begin{abstract}
Differentiation of the well-known addition theorem for Legendre polynomials produces results for sums over order $m$ of products of various derivatives of associated Legendre functions. The same method is applied to the corresponding addition theorems for vector and tensor spherical harmonics. Results are also given for Chebyshev polynomials of the second kind, corresponding to 'spin-weighted' associated Legendre functions, as used in studies of distributions of rotations.
\end{abstract}

\section{Introduction}

The addition theorem for Schmidt normalized associated Legendre functions, here denoted $P_{n}^{m}(\cos \theta)$ is given by Chapman and Bartels [1] as

$$
\sum_{m=0}^{n} P_{n}^{m}\left(\cos \theta_{1}\right) P_{n}^{m}\left(\cos \theta_{2}\right) \cos m\left(\phi_{1}-\phi_{2}\right)=P_{n}(\cos \Theta) .
$$

Schmidt normalized associated Legendre functions are defined in (50). The symbols $\theta_{1}$ and $\phi_{1}$ denote the colatitude and east longitude respectively, of a point $A$ on a spherical surface, whilst $\theta_{2}$ and $\phi_{2}$, are the corresponding quantities for another point $B$. The symbol $\Theta$ denotes angular length of the great circle arc between the two points $A\left(\theta_{1}, \phi_{1}\right)$ and $B\left(\theta_{2}, \phi_{2}\right)$.

The addition theorem plays an important part in potential theory and is widely used in a number of geophysical problems, such as geophysical exploration and the mathematical formulation of the theory of tides. Doodson [2] used the addition theorem as the basis of his work on the representation of the tide-producing potential. He used $C$ as the lunar hour angle in place of $\left(\phi_{1}-\phi_{2}\right)$. The lunar hour angle $C$

\footnotetext{
'Department of Applied Mathematics, University of Sydney, Sydney, 2006, Australia.

${ }^{2}$ Institute of Geophysics and Planetary Physics, Los Angeles, California, 90024, U.S.A.

(C) Australian Mathematical Society, 1995, Serial-fee code 0334-2700/95
} 


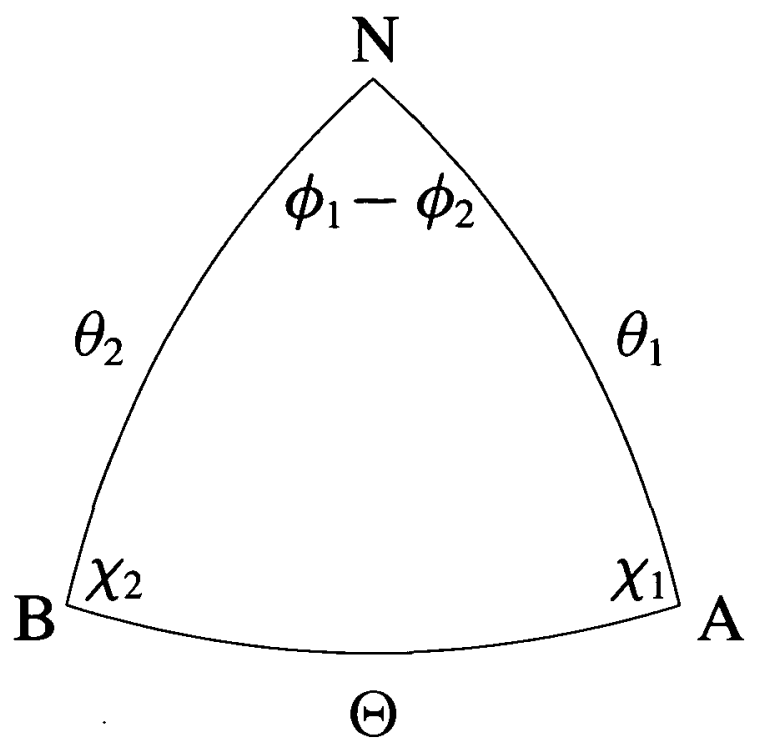

FIGURE 1. Spherical triangle showing nomenclature used.

increases at the rate of approximately $360^{\circ}$ per mean lunar day. The expansion of $P_{2}, P_{3}$ and $P_{4}$ in terms of $\cos C, \cos 2 C, \cos 3 C$ and $\cos 4 C$ by means of the addition theorem (1) was used to separate the so-called 'species of tidal constituents'.

In work on the weighted least squares analysis of geomagnetic field components $\mathrm{X}, \mathrm{Y}, \mathrm{Z}$, Whaler and Gubbins [7] required a number of sums over the order $m$ of the associated Legendre functions of degree $n$ and their derivatives. They report results obtained using an unpublished method suggested to them by P. H. Roberts, involving differentiation of the addition theorem (1) with respect to the parameters $\theta_{1}, \phi_{1}, \theta_{2}$ and $\phi_{2}$.

Vector and tensor spherical harmonics given in terms of unit normalised surface spherical harmonics $Y_{n}^{m}(\theta, \phi)$ have been defined in such a way as to satisfy addition theorems in vector and tensor forms.

In studies of distributions of rotations, the associated Chebyshev functions are the relevant orthogonal polynomials. They are very closely related to associated Legendre functions of half-odd integer degree and order, and they also satisfy an addition theorem. Results for multiple derivatives of this addition theorem are given. The results include as special cases the spherical trigonometry of hyperspheres used in dealing with combinations of rotations where a rotation about an axis through a given angle corresponds to a point on the hypersphere. 


\section{Derivatives of $\Theta, \chi_{1}, \chi_{2}$}

Expressions for the partial derivatives of $\Theta$ with respect to one of the parameters $\theta_{1}, \phi_{1}, \theta_{2}$ or $\phi_{2}$, are obtained by differentiating the formula known as the cosine rule of spherical trigonometry

$$
\cos \Theta=\cos \theta_{1} \cos \theta_{2}+\sin \theta_{1} \sin \theta_{2} \cos \left(\phi_{1}-\phi_{2}\right),
$$

which is the $n=1$ case of the addition theorem (1). By making use of the well-known results from spherical trigonometry

$$
\begin{aligned}
\sin \theta_{1} \cos \theta_{2}-\cos \theta_{1} \sin \theta_{2} \cos \left(\phi_{1}-\phi_{2}\right) & =\sin \Theta \cos \chi_{1}, \\
\cos \theta_{1} \sin \theta_{2}-\sin \theta_{1} \cos \theta_{2} \cos \left(\phi_{1}-\phi_{2}\right) & =\sin \Theta \cos \chi_{2}, \\
\sin \theta_{2} \sin \left(\phi_{1}-\phi_{2}\right) & =\sin \Theta \sin \chi_{1}, \\
\sin \theta_{1} \sin \left(\phi_{1}-\phi_{2}\right) & =\sin \Theta \sin \chi_{2},
\end{aligned}
$$

the following results for partial derivatives of $\Theta$ are obtained:

$$
\begin{array}{ll}
\frac{\partial \Theta}{\partial \theta_{1}}=\cos \chi_{1}, & \frac{\partial \Theta}{\partial \theta_{2}}=\cos \chi_{2}, \\
\frac{\partial \Theta}{\partial \phi_{1}}=\sin \theta_{1} \sin \chi_{1}, & \frac{\partial \Theta}{\partial \phi_{2}}=-\sin \theta_{2} \sin \chi_{2} .
\end{array}
$$

Derivatives of $\chi_{1}$ and $\chi_{2}$ with respect to $\theta_{1}$ and $\theta_{2}$ are obtained by differentiating (3) and (4) with respect to $\theta_{1}$ and $\theta_{2}$, making use of the cosine rule (2) and the result from spherical trigonometry

$$
\sin \theta_{1} \sin \theta_{2}+\cos \theta_{1} \cos \theta_{2} \cos \left(\phi_{1}-\phi_{2}\right)=\sin \chi_{1} \sin \chi_{2}-\cos \chi_{1} \cos \chi_{2} \cos \Theta,
$$

in which the right-hand side is simply the polar form of the left-hand side. Thus

$$
\begin{array}{ll}
\frac{\partial \chi_{1}}{\partial \theta_{1}}=-\sin \chi_{1} \cot \Theta, & \frac{\partial \chi_{1}}{\partial \theta_{2}}=\frac{\sin \chi_{2}}{\sin \Theta}, \\
\frac{\partial \chi_{2}}{\partial \theta_{1}}=\frac{\sin \chi_{1}}{\sin \Theta}, & \frac{\partial \chi_{2}}{\partial \theta_{2}}=-\sin \chi_{2} \cot \Theta .
\end{array}
$$

Derivatives of $\chi_{1}$ and $\chi_{2}$, with respect to $\phi_{1}$ and $\phi_{2}$, are obtained by differentiating (3) and (4) with respect to $\phi_{1}$ and $\phi_{2}$ and making use of the results

$$
\begin{aligned}
\sin \Theta \cos \theta_{1}-\cos \Theta \sin \theta_{1} \cos \chi_{1} & =\sin \theta_{2} \cos \chi_{2}, \\
\cos \theta_{1} \sin \left(\phi_{1}-\phi_{2}\right) & =\sin \chi_{1} \cos \chi_{2}+\cos \chi_{1} \sin \chi_{2} \cos \Theta, \\
\cos \theta_{2} \sin \left(\phi_{1}-\phi_{2}\right) & =\cos \chi_{1} \sin \chi_{2}+\sin \chi_{1} \cos \chi_{2} \cos \Theta,
\end{aligned}
$$


to obtain

$$
\begin{array}{ll}
\frac{\partial \chi_{1}}{\partial \phi_{1}}=-\frac{\sin \theta_{2} \cos \chi_{2}}{\sin \Theta}, & \frac{\partial \chi_{1}}{\partial \phi_{2}}=\frac{\sin \theta_{2} \cos \chi_{2}}{\sin \Theta}, \\
\frac{\partial \chi_{2}}{\partial \phi_{1}}=-\frac{\sin \theta_{1} \cos \chi_{1}}{\sin \Theta}, & \frac{\partial \chi_{2}}{\partial \phi_{2}}=\frac{\sin \theta_{1} \cos \chi_{1}}{\sin \Theta} .
\end{array}
$$

\section{Derivatives of the addition theorem}

Differentiation of the addition theorem (1) with respect to the parameters $\theta_{1}$ and $\phi_{1}$, and use of the expressions for the derivatives of $\Theta, \chi_{1}$ and $\chi_{2}$, given in Section 2, gives the following addition theorems directly.

$$
\begin{aligned}
\sum_{m=0}^{n} \frac{d P_{n}^{m}\left(\cos \theta_{1}\right)}{d \theta_{1}} P_{n}^{m}\left(\cos \theta_{2}\right) \cos m\left(\phi_{1}-\phi_{2}\right) & =\frac{d P_{n}(\cos \Theta)}{d \Theta} \cos \chi_{1} \\
\sum_{m=1}^{n} \frac{m}{\sin \theta_{1}} P_{n}^{m}\left(\cos \theta_{1}\right) P_{n}^{m}\left(\cos \theta_{2}\right) \sin m\left(\phi_{1}-\phi_{2}\right) & =-\frac{d P_{n}(\cos \Theta)}{d \Theta} \sin \chi_{1}
\end{aligned}
$$

Another group of formulae can be obtained by differentiating (17) with respect to $\theta_{1}, \theta_{2}, \phi_{1}$ and $\phi_{2}$. The second derivative of the Legendre polynomials $P_{n}(\cos \Theta)$ that arises can be removed, if required, by making use of the Legendre equation

$$
\frac{d^{2} P_{n}(\cos \Theta)}{d \Theta^{2}}+\cot \Theta \frac{d P_{n}(\cos \Theta)}{d \Theta}+n(n+1) P_{n}(\cos \Theta)=0 .
$$

Thus

$$
\begin{gathered}
\sum_{m=0}^{n} \frac{d^{2} P_{n}^{m}\left(\cos \theta_{1}\right)}{d \theta_{1}^{2}} P_{n}^{m}\left(\cos \theta_{2}\right) \cos m\left(\phi_{1}-\phi_{2}\right) \\
=\frac{d^{2} P_{n}(\cos \Theta)}{d \Theta^{2}} \cos ^{2} \chi_{1}+\frac{d P_{n}(\cos \Theta)}{d \Theta} \sin ^{2} \chi_{1} \cot \Theta \\
\sum_{m=0}^{n} \frac{d P_{n}^{m}\left(\cos \theta_{1}\right)}{d \theta_{1}} \frac{d P_{n}^{m}\left(\cos \theta_{2}\right)}{d \theta_{2}} \cos m\left(\phi_{1}-\phi_{2}\right) \\
=\frac{d^{2} P_{n}(\cos \Theta)}{d \Theta^{2}} \cos \chi_{1} \cos \chi_{2}-\frac{d P_{n}(\cos \Theta)}{d \Theta} \frac{\sin \chi_{1} \sin \chi_{2}}{\sin \Theta} \\
\sum_{m=1}^{n} \frac{d P_{n}^{m}\left(\cos \theta_{1}\right)}{d \theta_{1}} \frac{m}{\sin \theta_{2}} P_{n}^{m}\left(\cos \theta_{2}\right) \sin m\left(\phi_{1}-\phi_{2}\right) \\
=-\frac{d^{2} P_{n}(\cos \Theta)}{d \Theta^{2}} \cos \chi_{1} \sin \chi_{2}-\frac{d P_{n}(\cos \Theta)}{d \Theta} \frac{\sin \chi_{1} \cos \chi_{2}}{\sin \Theta}
\end{gathered}
$$


Differentiate (18) with respect to $\theta_{1}, \phi_{2}$, to obtain

$$
\begin{gathered}
\sum_{m=1}^{n} \frac{d}{d \theta_{1}}\left[\frac{m}{\sin \theta_{1}} P_{n}^{m}\left(\cos \theta_{1}\right)\right] P_{n}^{m}\left(\cos \theta_{2}\right) \sin m\left(\phi_{1}-\phi_{2}\right) \\
=\left[-\frac{d^{2} P_{n}(\cos \Theta)}{d \Theta^{2}}+\cot \Theta \frac{d P_{n}(\cos \Theta)}{d \Theta}\right] \sin \chi_{1} \cos \chi_{1}, \\
\sum_{m=1}^{n} \frac{m}{\sin \theta_{1}} P_{n}^{m}\left(\cos \theta_{1}\right) \frac{m}{\sin \theta_{2}} P_{n}^{m}\left(\cos \theta_{2}\right) \cos m\left(\phi_{1}-\phi_{2}\right) \\
=-\frac{d^{2} P_{n}(\cos \Theta)}{d \Theta^{2}} \sin \chi_{1} \sin \chi_{2}+\frac{d P_{n}(\cos \Theta)}{d \Theta} \frac{\cos \chi_{1} \cos \chi_{2}}{\sin \Theta} .
\end{gathered}
$$

Despite their simple appearance, (17)-(23) are not easy to apply. One must first use (2)-(6) to evaluate $\chi_{1}, \chi_{2}$, and $\Theta$ for the given $\left(\theta_{1}, \phi_{1}\right)$ and $\left(\theta_{2}, \phi_{2}\right)$. Such complications do not arise in the special cases given in the following section.

\section{Special cases}

The sums given in Section 3 for the case $n=1$ are well known formulae of spherical trigonometry: (17) reduces to (3), (18) to (5), (19) to the addition theorem (1), (20) to (9) and (21) reduces to (13). Equation (23) reduces to the polar form of the cosine rule of spherical trigonometry

$$
\cos \left(\phi_{1}-\phi_{2}\right)=\sin \chi_{1} \sin \chi_{2} \cos \Theta-\cos \chi_{1} \cos \chi_{2} .
$$

In the special case that arises when $A$ lies on the same meridian as $B$ and to the north of it, then from Figure 1, the values of $\Theta, \chi_{1}, \chi_{2},\left(\phi_{1}-\phi_{2}\right)$, are given by

$$
\Theta=\theta_{2}-\theta_{1}, \quad \chi_{1}=\pi, \quad \chi_{2}=0, \quad \phi_{1}-\phi_{2}=0 .
$$

With the substitutions indicated in (25), then (1), (17), (19), (20), (23) give the following duplication formulae

$$
\begin{aligned}
\sum_{m=0}^{n} P_{n}^{m}\left(\cos \theta_{1}\right) P_{n}^{m}\left(\cos \theta_{2}\right) & =P_{n}\left[\cos \left(\theta_{2}-\theta_{1}\right)\right], \\
\sum_{m=0}^{n} \frac{d P_{n}^{m}\left(\cos \theta_{1}\right)}{d \theta_{1}} P_{n}^{m}\left(\cos \theta_{2}\right) & =-\left[\frac{d P_{n}(\cos \Theta)}{d \Theta}\right]_{\Theta=\theta_{2}-\theta_{1}}, \\
\sum_{m=0}^{n} \frac{d^{2} P_{n}^{m}\left(\cos \theta_{1}\right)}{d \theta_{1}{ }^{2}} P_{n}^{m}\left(\cos \theta_{2}\right) & =\left[\frac{d^{2} P_{n}(\cos \Theta)}{d \Theta^{2}}\right]_{\Theta=\theta_{2}-\theta_{1}}, \\
\sum_{m=0}^{n} \frac{d P_{n}^{m}\left(\cos \theta_{1}\right)}{d \theta_{1}} \frac{d P_{n}^{m}\left(\cos \theta_{2}\right)}{d \theta_{2}} & =-\left[\frac{d^{2} P_{n}(\cos \Theta)}{d \Theta^{2}}\right]_{\Theta=\theta_{2}-\theta_{1}},
\end{aligned}
$$




$$
\sum_{m=1}^{n} \frac{m}{\sin \theta_{1}} P_{n}^{m}\left(\cos \theta_{1}\right) \frac{m}{\sin \theta_{2}} P_{n}^{m}\left(\cos \theta_{2}\right)=-\left[\frac{1}{\sin \Theta} \frac{d P_{n}(\cos \Theta)}{d \Theta}\right]_{\Theta=\theta_{2}-\theta_{1}} .
$$

These results have been used in spherical harmonic analysis of the geomagnetic main field as a linear inverse problem (Whaler and Gubbins [7]), and in the theory of off-centre or eccentric geomagnetic dipoles, for example Elsasser [3]. In the further special case $\Theta=0$, when $A$ and $B$ coincide, $\theta_{1}=\theta_{2}=\theta$, the results

$$
\begin{array}{rlrl}
{\left[P_{n}(\cos \Theta)\right]_{\Theta=0}} & =1, & {\left[\frac{1}{\sin \Theta} \frac{d P_{n}(\cos \Theta)}{d \Theta}\right]_{\Theta=0}} & =-\frac{1}{2} n(n+1), \\
{\left[\frac{d P_{n}(\cos \Theta)}{d \Theta}\right]_{\Theta=0}=0,} & {\left[\frac{d^{2} P_{n}(\cos \Theta)}{d \Theta^{2}}\right]_{\Theta=0}=-\frac{1}{2} n(n+1),}
\end{array}
$$

together with (26)-(30), give the following sums

$$
\begin{aligned}
\sum_{m=0}^{n}\left[P_{n}^{m}(\cos \theta)\right]^{2} & =1, \\
\sum_{m=0}^{n} \frac{d P_{n}^{m}(\cos \theta)}{d \theta} P_{n}^{m}(\cos \theta) & =0, \\
\sum_{m=0}^{n} \frac{d^{2} P_{n}^{m}(\cos \theta)}{d \theta^{2}} P_{n}^{m}(\cos \theta) & =-\frac{1}{2} n(n+1), \\
\sum_{m=0}^{n}\left[\frac{d P_{n}^{m}(\cos \theta)}{d \theta}\right]^{2} & =\frac{1}{2} n(n+1), \\
\sum_{m=1}^{n}\left[\frac{m}{\sin \theta} P_{n}^{m}(\cos \theta)\right]^{2} & =\frac{1}{2} n(n+1) .
\end{aligned}
$$

Equation (34) could also have been derived from (33) by differentiation.

A number of other special cases involving sums of higher derivatives are easily obtained and are included here for completeness. In the special case that arises when $A$ lies on the same meridian as $B$ and the constraints of (25) apply, differentiate (19) twice with respect to $\theta_{2}$ to obtain

$$
\sum_{m=0}^{n} \frac{d^{2} P_{n}^{m}\left(\cos \theta_{1}\right)}{d \theta_{1}^{2}} \frac{d^{2} P_{n}^{m}\left(\cos \theta_{2}\right)}{d \theta_{2}^{2}}=\left[\frac{d^{4} P_{n}(\cos \Theta)}{d \Theta^{4}}\right]_{\Theta=\theta_{2}-\theta_{1}}
$$

Differentiate (22) with respect to $\phi_{2}$ and $\theta_{2}$ to obtain

$$
\begin{gathered}
\sum_{m=1}^{n} \frac{d}{d \theta_{1}}\left[\frac{m}{\sin \theta_{1}} P_{n}^{m}\left(\cos \theta_{1}\right)\right] \frac{d}{d \theta_{2}}\left[\frac{m}{\sin \theta_{2}} P_{n}^{m}\left(\cos \theta_{2}\right)\right] \\
=\left\{\frac{d^{2}}{d \Theta^{2}}\left[\frac{1}{\sin \Theta} \frac{d P_{n}(\cos \Theta)}{d \Theta}\right]\right\}_{\Theta=\theta_{2}-\theta_{1}} .
\end{gathered}
$$


In the further special case when $\Theta=0$, when $A$ and $B$ coincide, then $\theta_{1}=\theta_{2}=\theta$, then the two previous equations become

$$
\begin{aligned}
\sum_{m=0}^{n}\left[\frac{d^{2} P_{n}^{m}(\cos \theta)}{d \theta^{2}}\right]^{2} & =\left[\frac{d^{4} P_{n}(\cos \Theta)}{d \Theta^{4}}\right]_{\Theta=0}, \\
& =\frac{1}{8} n(n+1)\left(3 n^{2}+3 n-2\right),
\end{aligned}
$$

and

$$
\begin{aligned}
\sum_{m=1}^{n}\left\{\frac{d}{d \theta}\left[\frac{m}{\sin \theta} P_{n}^{m}(\cos \theta)\right]\right\}^{2} & =\left\{\frac{d^{2}}{d \Theta^{2}}\left[\frac{1}{\sin \Theta} \frac{d P_{n}(\cos \Theta)}{d \Theta}\right]\right\}_{\Theta=0} \\
& =\frac{1}{8}(n-1) n(n+1)(n+2) .
\end{aligned}
$$

Not all such sums are independent of $\theta$, as are the results of (33)-(39). To establish the two sums given in (44) and (45) below, one proceeds as follows.

The derivative of (36) with respect to $\theta$ gives

$$
\sum_{m=0}^{n} \frac{d^{2} P_{n}^{m}(\cos \theta)}{d \theta^{2}} \frac{d P_{n}^{m}(\cos \theta)}{d \theta}=0 .
$$

Differentiate (37) with respect to $\theta$ to obtain

$$
\sum_{m=1}^{n} \frac{m^{2}}{\sin ^{2} \theta} \frac{d P_{n}^{m}(\cos \theta)}{d \theta} P_{n}^{m}(\cos \theta)=\frac{1}{2} n(n+1) \frac{\cos \theta}{\sin \theta} .
$$

Given the differential equation for associated Legendre functions $P_{n}^{m}(\cos \theta)$ in the form

$$
\frac{d^{2} P_{n}^{m}}{d \theta^{2}}+\cot \theta \frac{d P_{n}^{m}}{d \theta}+n(n+1) P_{n}^{m}-\frac{m^{2}}{\sin ^{2} \theta} P_{n}^{m}=0,
$$

multiply throughout by $d^{2} P_{n}^{m} / d \theta^{2}$ and sum over $m$, making use of (38), (40), (35), to obtain

$$
\sum_{m=1}^{n} \frac{m^{2}}{\sin ^{2} \theta} \frac{d^{2} P_{n}^{m}(\cos \theta)}{d \theta^{2}} P_{n}^{m}(\cos \theta)=-\frac{1}{8} n(n+1)\left(n^{2}+n+2\right) .
$$

Differentiate (41) with respect to $\theta$ and use the sum in (43) to obtain

$$
\sum_{m=1}^{n}\left[\frac{m}{\sin \theta} \frac{d P_{n}^{m}(\cos \theta)}{d \theta}\right]^{2}=\frac{\frac{1}{2} n(n+1)}{\sin ^{2} \theta}+\frac{1}{8} n(n+1)(n-2)(n+3) .
$$

Multiply the differential equation (42) throughout by $m^{2} P_{n}^{m}(\cos \theta) / \sin ^{2} \theta$ and sum over $m$, making use of (37), (41) and (43), to obtain

$$
\sum_{m=1}^{n}\left[\frac{m^{2}}{\sin ^{2} \theta} P_{n}^{m}(\cos \theta)\right]^{2}=\frac{\frac{1}{2} n(n+1)}{\sin ^{2} \theta}+\frac{3}{8}(n-1) n(n+1)(n+2) .
$$


The derivatives required in (31), (32), (38) and (39) are obtained by expanding the Rodrigues's formula for Legendre polynomials in ascending powers of $(1-\mu)$, where $\mu=\cos \theta$.

$$
P_{n}(\mu)=\sum_{r=0}^{n} \frac{(n+r) !}{2^{r}(n-r) ! r ! r !}(\mu-1)^{r}={ }_{2} F_{1}\left(-n, n+1 ; 1 ; \frac{1}{2}(1-\mu)\right) .
$$

It follows from (46) that the $r^{\text {th }}$ derivative of the Legendre polynomials $P_{n}(\mu)$ evaluated at $\mu=1$ is given by

$$
\left[\left(\frac{d}{d \mu}\right)^{r} P_{n}(\mu)\right]_{\mu=1}=P_{n}^{(r)}(1)=\frac{(n+r) !}{2^{r}(n-r) ! r !},
$$

with special cases:

$$
P_{n}(1)=1, \quad P_{n}^{\prime}(1)=\frac{1}{2} n(n+1), \quad P_{n}^{\prime \prime}(1)=\frac{1}{8}(n-1) n(n+1)(n+2) .
$$

Multiple derivatives of $P_{n}(\cos \theta)$ with respect to $\theta$ at $\theta=0$ are obtained using the chain rule; the odd order derivatives are found to be zero, and the first few even order derivatives are

$$
\begin{aligned}
{\left[P_{n}(\cos \theta)\right]_{\theta=0} } & =1, \\
& {\left[\frac{d^{2} P_{n}(\cos \theta)}{d \theta^{2}}\right]_{\theta=0}=-P_{n}^{\prime}(1), } \\
{\left[\frac{d^{4} P_{n}(\cos \theta)}{d \theta^{4}}\right]_{\theta=0} } & =3 P_{n}^{\prime \prime}(1)+P_{n}^{\prime}(1), \\
{\left[\frac{d^{6} P_{n}(\cos \theta)}{d \theta^{6}}\right]_{\theta=0} } & =-15 P_{n}^{\prime \prime \prime}(1)-15 P_{n}^{\prime \prime}(1)-P_{n}^{\prime}(1) .
\end{aligned}
$$

\section{Vector spherical harmonics}

The sums given in Sections 3 and 4 have a simple form because of the use of Schmidt normalised associated Legendre functions, where

$$
P_{n}^{m}(\mu)=\frac{1}{2^{n} n !} \sqrt{\left(2-\delta_{m}^{0}\right) \frac{(n-m) !}{(n+m) !}}\left(1-\mu^{2}\right)^{m / 2}\left(\frac{d}{d \mu}\right)^{n+m}\left(\mu^{2}-1\right)^{n},
$$

which is valid for positive and negative values of $m$ such that $|m| \leq n$. Since $P_{-n-1}^{m}(\mu)=P_{n}^{m}(\mu)$, it is usual to assume that $n \geq 0$. 
The result that

$$
\begin{aligned}
(n+m) !\left(1-\mu^{2}\right)^{-m / 2} & \left(\frac{d}{d \mu}\right)^{n-m}\left(\mu^{2}-1\right)^{n} \\
& =(-1)^{m}(n-m) !\left(1-\mu^{2}\right)^{m / 2}\left(\frac{d}{d \mu}\right)^{n+m}\left(\mu^{2}-1\right)^{n},
\end{aligned}
$$

is easily verified by using Leibnitz's theorem on the multiple derivatives of a product of two functions and rearranging the factorial terms that arise. It then follows directly that, for Schmidt normalised functions,

$$
P_{n}^{-m}(\mu)=(-1)^{m} P_{n}^{m}(\mu),
$$

and further, that the addition theorem sum

$$
\begin{aligned}
\sum_{m=0}^{n} P_{n}^{m}\left(\cos \theta_{1}\right) & P_{n}^{m}\left(\cos \theta_{2}\right) \cos m\left(\phi_{1}-\phi_{2}\right) \\
& =\sum_{m=-n}^{n} P_{n, m}\left(\cos \theta_{1}\right) P_{n, m}\left(\cos \theta_{2}\right) e^{-i m\left(\phi_{1}-\phi_{2}\right)} \\
& =\sum_{m=-n}^{n} \overline{Y_{n}^{m}\left(\theta_{1}, \phi_{1}\right)} Y_{n}^{m}\left(\theta_{2}, \phi_{2}\right)
\end{aligned}
$$

where $Y_{n}^{m}(\theta, \phi)=P_{n, m}(\cos \theta) e^{i m \phi}$, and

$$
P_{n, m}(\mu)=\frac{1}{2^{n} n !} \sqrt{\frac{(n-m) !}{(n+m) !}}\left(1-\mu^{2}\right)^{m / 2}\left(\frac{d}{d \mu}\right)\left(\mu^{2}-1\right)^{n} .
$$

The addition theorem for associated Legendre functions becomes

$$
\sum_{m=-n}^{n} \overline{Y_{n}^{m}\left(\theta_{1}, \phi_{1}\right)} Y_{n}^{m}\left(\theta_{2}, \phi_{2}\right)=(2 n+1) P_{n}(\cos \Theta),
$$

where the overline denotes a complex conjugate.

In view of the treatment of addition theorems for vector and tensor spherical harmonics to follow, it is convenient to set down explicitly the addition theorems obtained by differentiating (52).

$$
\begin{aligned}
& \sum_{m=-n}^{n} \frac{\partial \overline{Y_{n}^{m}\left(\theta_{1}, \phi_{1}\right)}}{\partial \theta_{1}} Y_{n}^{m}\left(\theta_{2}, \phi_{2}\right)=(2 n+1) \frac{d P_{n}(\cos \Theta)}{d \Theta} \cos \chi_{1}, \\
& \sum_{m=-n}^{n} \frac{1}{\sin \theta_{1}} \frac{\partial \overline{Y_{n}^{m}\left(\theta_{1}, \phi_{1}\right)}}{\partial \phi_{1}} Y_{n}^{m}\left(\theta_{2}, \phi_{2}\right)=(2 n+1) \frac{d P_{n}(\cos \Theta)}{d \Theta} \sin \chi_{1},
\end{aligned}
$$




$$
\begin{aligned}
& \sum_{m=-n}^{n} \frac{\partial^{2} \overline{Y_{n}^{m}\left(\theta_{1}, \phi_{1}\right)}}{\partial \theta_{1}^{2}} Y_{n}^{m}\left(\theta_{2}, \phi_{2}\right) \\
& =(2 n+1)\left[\frac{d^{2} P_{n}(\cos \Theta)}{d \Theta^{2}} \cos ^{2} \chi_{1}+\frac{d P_{n}(\cos \Theta)}{d \Theta} \sin ^{2} \chi_{1} \cot \Theta\right] \text {, } \\
& \sum_{m=-n}^{n} \frac{\partial \overline{Y_{n}^{m}\left(\theta_{1}, \phi_{1}\right)}}{\partial \theta_{1}} \frac{\partial Y_{n}^{m}\left(\theta_{2}, \phi_{2}\right)}{\partial \theta_{2}} \\
& =(2 n+1)\left[\frac{d^{2} P_{n}(\cos \Theta)}{d \Theta^{2}} \cos \chi_{1} \cos \chi_{2}-\frac{d P_{n}(\cos \Theta)}{d \Theta} \frac{\sin \chi_{1} \sin \chi_{2}}{\sin \Theta}\right], \\
& \sum_{m=-n}^{n} \frac{\partial \overline{Y_{n}^{m}\left(\theta_{1}, \phi_{1}\right)}}{\partial \theta_{1}} \frac{1}{\sin \theta_{2}} \frac{\partial Y_{n}^{m}\left(\theta_{2}, \phi_{2}\right)}{\partial \phi_{2}} \\
& =-(2 n+1)\left[\frac{d^{2} P_{n}(\cos \Theta)}{d \Theta^{2}} \cos \chi_{1} \sin \chi_{2}+\frac{d P_{n}(\cos \Theta)}{d \Theta} \frac{\sin \chi_{1} \cos \chi_{2}}{\sin \Theta}\right] \text {, } \\
& \sum_{m=-n}^{n} \frac{d}{d \theta_{1}}\left[\frac{1}{\sin \theta_{1}} \frac{\partial \overline{Y_{n}^{m}\left(\theta_{1}, \phi_{1}\right)}}{\partial \phi_{1}}\right] Y_{n}^{m}\left(\theta_{2}, \phi_{2}\right) \\
& =(2 n+1)\left[\frac{d^{2} P_{n}(\cos \Theta)}{d \Theta^{2}}-\frac{d P_{n}(\cos \Theta)}{d \Theta} \cot \Theta\right] \cos \chi_{1} \sin \chi_{1}, \\
& \sum_{m=-n}^{n} \frac{1}{\sin \theta_{1}} \frac{\partial}{\partial \phi_{1}}\left[\frac{\partial \overline{Y_{n}^{m}\left(\theta_{1}, \phi_{1}\right)}}{\partial \theta_{1}}\right] Y_{n}^{m}\left(\theta_{2}, \phi_{2}\right) \\
& =(2 n+1)\left[\frac{d^{2} P_{n}(\cos \Theta)}{d \Theta^{2}} \cos \chi_{1} \sin \chi_{1}+\frac{d P_{n}(\cos \Theta)}{d \Theta} \frac{\sin ^{2} \chi_{1} \cot \chi_{2}}{\sin \Theta}\right] \text {, } \\
& \sum_{m=-n}^{n} \frac{1}{\sin \theta_{1}} \frac{\partial \overline{Y_{n}^{m}\left(\theta_{1}, \phi_{1}\right)}}{\partial \phi_{1}} \frac{1}{\sin \theta_{2}} \frac{\partial Y_{n}^{m}\left(\theta_{2}, \phi_{2}\right)}{\partial \phi_{2}} \\
& =(2 n+1)\left[-\frac{d^{2} P_{n}(\cos \Theta)}{d \Theta^{2}} \sin \chi_{1} \sin \chi_{2}+\frac{d P_{n}(\cos \Theta)}{d \Theta} \frac{\cos \chi_{1} \cos \chi_{2}}{\sin \Theta}\right] \text {, } \\
& \sum_{m=-n}^{n} \frac{1}{\sin ^{2} \theta_{1}} \frac{\partial^{2} \overline{Y_{n}^{m}\left(\theta_{1}, \phi_{1}\right)}}{\partial \phi_{1}^{2}} Y_{n}^{m}\left(\theta_{2}, \phi_{2}\right) \\
& =(2 n+1)\left[\frac{d^{2} P_{n}(\cos \Theta)}{d \Theta^{2}} \sin ^{2} \chi_{1}-\frac{d P_{n}(\cos \Theta)}{d \Theta} \frac{\sin \chi_{1} \cos \chi_{1}}{\sin \Theta} \cot \chi_{2}\right] \text {, }
\end{aligned}
$$

where (59) and (61) are obtained by differentiating (53) and (54) with respect to $\phi_{1}$.

Vector (surface) spherical harmonics $\mathbf{Y}_{1, n}^{m}(\theta, \phi), \mathbf{Y}_{0, n}^{m}(\theta, \phi), \mathbf{Y}_{-1, n}^{m}(\theta, \phi)$, are defined by 


$$
\begin{aligned}
\mathbf{Y}_{1, n}^{m}(\theta, \phi) & =r^{n+2} \nabla\left[r^{-n-1} Y_{n}^{m}(\theta, \phi)\right] / \sqrt{(n+1)(2 n+1)}, \\
\mathbf{Y}_{0, n}^{m}(\theta, \phi) & =\mathbf{L} Y_{n}^{m}(\theta, \phi) / \sqrt{n(n+1)}, \\
\mathbf{Y}_{-1, n}^{m}(\theta, \phi) & =r^{1-n} \nabla\left[r^{n} Y_{n}^{m}(\theta, \phi)\right] / \sqrt{n(2 n+1)},
\end{aligned}
$$

where the angular momentum operator $\mathbf{L}=-i \mathbf{r} \times \nabla$. The spherical polar components of the vector spherical harmonics are given by

$$
\begin{aligned}
& \mathbf{Y}_{1, n}^{m}(\theta, \phi)=\frac{1}{\sqrt{(n+1)(2 n+1)}}\left[-(n+1) Y_{n}^{m}(\theta, \phi) \mathbf{e}_{r}\right. \\
& \left.\quad+\frac{\partial Y_{n}^{m}(\theta, \phi)}{\partial \theta} \mathbf{e}_{\theta}+\frac{1}{\sin \theta} \frac{\partial Y_{n}^{m}(\theta, \phi)}{\partial \phi} \mathbf{e}_{\phi}\right] \\
& \mathbf{Y}_{0, n}^{m}(\theta, \phi)=\frac{i}{\sqrt{n(n+1)}}\left[\frac{1}{\sin \theta} \frac{\partial Y_{n}^{m}(\theta, \phi)}{\partial \phi} \mathbf{e}_{\theta}-\frac{\partial Y_{n}^{m}(\theta, \phi)}{\partial \theta} \mathbf{e}_{\phi}\right], \\
& \mathbf{Y}_{-1, n}^{m}(\theta, \phi) \\
& =\frac{1}{\sqrt{n(2 n+1)}}\left[n Y_{n}^{m}(\theta, \phi) \mathbf{e}_{r}+\frac{\partial Y_{n}^{m}(\theta, \phi)}{\partial \theta} \mathbf{e}_{\theta}+\frac{1}{\sin \theta} \frac{\partial Y_{n}^{m}(\theta, \phi)}{\partial \phi} \mathbf{e}_{\phi}\right] .
\end{aligned}
$$

These vector spherical harmonics are more commonly denoted by $\mathbf{Y}_{n, n+1}^{m}(\theta, \phi)$, $\mathbf{Y}_{n, n}^{m}(\theta, \phi), \mathbf{Y}_{n, n-1}^{m}(\theta, \phi)$, respectively, but this notation does not give enough emphasis to the property that these vector spherical harmonics transform under rotation of the reference frame with the same transformation law as for the scalar spherical harmonics $Y_{n}^{m}(\theta, \phi)$. In the context of geomagnetism these vector spherical harmonics are very well known. $\mathbf{Y}_{1, n}^{m}(\theta, \phi)$ is a component of the magnetic field originating within the Earth evaluated on a sphere of unit radius; $\mathbf{Y}_{0, n}^{m}(\theta, \phi)$ is a component of the so-called nonpotential field being the magnetic field associated with Earth-to-air electric currents, and $\mathbf{Y}_{-1, n}^{m}(\theta, \phi)$ is a component of the magnetic field originating outside the Earth.

Many of the properties of the vector spherical harmonics are most easily derived when they are expressed in Cartesian components, or better still, in terms of complex reference vectors $\mathbf{e}_{1}, \mathbf{e}_{0}, \mathbf{e}_{-1}$, defined by

$$
\mathbf{e}_{1}=-\frac{1}{\sqrt{2}}\left(\mathbf{e}_{x}+i \mathbf{e}_{y}\right), \quad \mathbf{e}_{0}=\mathbf{e}_{z}, \quad \mathbf{e}_{-1}=\frac{1}{\sqrt{2}}\left(\mathbf{e}_{x}-i \mathbf{e}_{y}\right),
$$

which have the orthogonality property

$$
\overline{\mathbf{e}}_{\mu} \cdot \mathbf{e}_{\nu}=\delta_{\mu \nu}, \quad(\mu, \nu=-1,0,1) .
$$

A vector $\mathbf{B}$ say, with Cartesian components $\left(B_{x}, B_{y}, B_{z}\right)$, will have a complex reference vector form

$$
\mathbf{B}=-B_{1} \mathbf{e}_{-1}+B_{0} \mathbf{e}_{0}-B_{-1} \mathbf{e}_{-1},
$$


where the complex reference components are given by $B_{\mu}=\mathbf{B} \cdot \mathbf{e}_{\mu}$ for $\mu=-1,0,1$. The complex reference components of the gradient operator $\nabla$ and the angular momentum operator $\mathbf{L}=-i \mathbf{r} \times \nabla$ are given by

$$
\begin{aligned}
\nabla_{1} & =-\frac{1}{\sqrt{2}}\left(\frac{\partial}{\partial x}+i \frac{\partial}{\partial y}\right)=-\frac{1}{\sqrt{2}} e^{i \phi}\left(\sin \theta \frac{\partial}{\partial r}+\frac{\cos \theta}{r} \frac{\partial}{\partial \theta}+\frac{i}{r \sin \theta} \frac{\partial}{\partial \phi}\right) \\
\nabla_{0} & =\frac{\partial}{\partial z}=\cos \theta \frac{\partial}{\partial r}-\frac{\sin \theta}{r} \frac{\partial}{\partial \theta} \\
\nabla_{-1} & =\frac{1}{\sqrt{2}}\left(\frac{\partial}{\partial x}-i \frac{\partial}{\partial y}\right)=\frac{1}{\sqrt{2}} e^{-i \phi}\left(\sin \theta \frac{\partial}{\partial r}+\frac{\cos \theta}{r} \frac{\partial}{\partial \theta}-\frac{i}{r \sin \theta} \frac{\partial}{\partial \phi}\right) \\
L_{1} & =-\frac{1}{\sqrt{2}}\left(L_{x}+i L_{y}\right)=-\frac{1}{\sqrt{2}} e^{i \phi}\left(\frac{\partial}{\partial \theta}+i \cot \theta \frac{\partial}{\partial \phi}\right) \\
L_{0} & =L_{z}=-i \frac{\partial}{\partial \phi} \\
L_{-1} & =\frac{1}{\sqrt{2}}\left(L_{x}-i L_{y}\right)=\frac{1}{\sqrt{2}} e^{-i \phi}\left(\frac{\partial}{\partial \theta}-i \cot \theta \frac{\partial}{\partial \phi}\right) .
\end{aligned}
$$

Expressions for the vector spherical harmonics in terms of complex reference vectors are

$$
\begin{gathered}
\mathbf{Y}_{1, n}^{m}(\theta, \phi)=\frac{1}{\sqrt{(n+1)(2 n+3)}}\left[\sqrt{(n+m+1)(n+m+2) / 2} Y_{n+1}^{m+1}(\theta, \phi) \mathbf{e}_{-1}\right. \\
-\sqrt{(n-m+1)(n+m+1)} Y_{n+1}^{m}(\theta, \phi) \mathbf{e}_{0} \\
\left.+\sqrt{(n-m+1)(n-m+2) / 2} Y_{n+1}^{m-1}(\theta, \phi) \mathbf{e}_{1}\right] \\
\mathbf{Y}_{0, n}^{m}(\theta, \phi)=\frac{1}{\sqrt{n(n+1)}}\left[\sqrt{(n+m+1)(n-m) / 2} Y_{n}^{m+1}(\theta, \phi) \mathbf{e}_{-1}\right. \\
\left.+m Y_{n}^{m}(\theta, \phi) \mathbf{e}_{0}-\sqrt{(n+m)(n-m+1) / 2} Y_{n}^{m-1}(\theta, \phi) \mathbf{e}_{1}\right] \\
\mathbf{Y}_{-1, n}^{m}(\theta, \phi)=\frac{1}{\sqrt{n(2 n-1)}}\left[\sqrt{(n-m-1)(n-m) / 2} Y_{n-1}^{m+1}(\theta, \phi) \mathbf{e}_{-1}\right. \\
+\sqrt{(n+m)(n-m)} Y_{n-1}^{m}(\theta, \phi) \mathbf{e}_{0} \\
\left.+\sqrt{(n+m-1)(n+m) / 2} Y_{n-1}^{m-1}(\theta, \phi) \mathbf{e}_{1}\right]
\end{gathered}
$$

and a single formula equivalent to (69) can be given using $3-j$ coefficients to "couple" together scalar spherical harmonics $Y_{n}^{m}(\theta, \phi)$ and complex reference vectors $\mathrm{e}_{1}, \mathbf{e}_{0}$, 
$\mathbf{e}_{-1}$. Thus for $v=-1,0,1$,

$$
\mathbf{Y}_{\nu, n}^{m}(\theta, \phi)=(-1)^{n-m} \sqrt{2 n+1} \sum_{\mu=-1}^{1}\left(\begin{array}{ccc}
n & n+\nu & 1 \\
m & -m+\mu & -\mu
\end{array}\right) Y_{n+\nu}^{m-\mu}(\theta, \phi) \mathbf{e}_{\mu} .
$$

The triangle rule for nonzero $3-j$ coefficients requires that the parameters $n, n+\nu, 1$ should be able to form a triangle, and indicates that only three vector spherical harmonics as given in (69) are defined, corresponding to $v=1,0,-1$.

Vector spherical harmonics transform like scalar surface spherical harmonics under rotation of the reference frame and satisfy the orthogonality condition

$$
\frac{1}{4 \pi} \int_{0}^{2 \pi} \int_{0}^{\pi} \overline{\mathbf{Y}_{\mu, n}^{m}(\theta, \phi)} \cdot \mathbf{Y}_{\nu, N}^{M}(\theta, \phi) \sin \theta d \theta d \phi=\delta_{n}^{N} \delta_{m}^{M} \delta_{\mu}^{\nu}
$$

The expressions given in (69) can be used to show directly that vector spherical harmonics satisfy the addition theorem

$$
\sum_{m=-n}^{n} \overline{\mathbf{Y}_{v, n}^{m}\left(\theta_{1}, \phi_{1}\right)} d \cdot \mathbf{Y}_{v, n}^{m}\left(\theta_{2}, \phi_{2}\right)=(2 n+1) P_{n+v}(\cos \Theta), \quad v=-1,0,1,
$$

and also satisfy a tensor form requiring a sum of direct products over the degree $n$,

$$
\sum_{\zeta=-1}^{1} \sum_{m=-n}^{n} \overline{\mathbf{Y}_{\zeta, n+v}^{m}\left(\theta_{1}, \phi_{1}\right)} \mathbf{Y}_{\zeta, n+v}^{m}\left(\theta_{2}, \phi_{2}\right)=(2 n+2 v+1) P_{n+\nu}(\cos \Theta) U,
$$

where $U$ is the unit tensor

$$
U=\mathbf{e}_{x} \mathbf{e}_{x}+\mathbf{e}_{y} \mathbf{e}_{y}+\mathbf{e}_{z} \mathbf{e}_{z}=\overline{\mathbf{e}_{1}} \mathbf{e}_{1}+\overline{\mathbf{e}_{0}} \mathbf{e}_{0}+\overline{\mathbf{e}_{-1}} \mathbf{e}_{-1} .
$$

The addition theorems (72) and (73) are very difficult to verify directly using the spherical polar forms for vector spherical harmonics, because of the need to evaluate all scalar products such as $\mathbf{e}_{\theta_{1}} \cdot \mathbf{e}_{\theta_{2}}$. The relative simplicity of proofs of (72) and (73), based on the Cartesian forms given in (69) or the equivalent $3-j$ coefficient form in (70) may persuade the reader of the merit in using Cartesian expressions when working with spherical harmonics. Differentiation of the addition theorem in (72) produces the vector analogues of (53)-(60)

$$
\begin{aligned}
& \sum_{m=-n}^{n} \frac{\partial \overline{\mathbf{Y}_{v, n}^{m}\left(\theta_{1}, \phi_{1}\right)}}{\partial \theta_{1}} \cdot \mathbf{Y}_{v, n}^{m}\left(\theta_{2}, \phi_{2}\right)=(2 n+1) \frac{d P_{n+v}(\cos \Theta)}{d \Theta} \cos \chi_{1}, \\
& \sum_{m=-n}^{n} \frac{1}{\sin \theta_{1}} \frac{\partial \overline{\mathbf{Y}_{v, n}^{m}\left(\theta_{1}, \phi_{1}\right)}}{\partial \phi_{1}} \cdot \mathbf{Y}_{v, n}^{m}\left(\theta_{2}, \phi_{2}\right)=(2 n+1) \frac{d P_{n+v}(\cos \Theta)}{d \Theta} \sin \chi_{1}
\end{aligned}
$$




$$
\begin{aligned}
& \sum_{m=-n}^{n} \frac{\partial^{2} \overline{\mathbf{Y}_{v, n}^{m}\left(\theta_{1}, \phi_{1}\right)}}{\partial \theta_{1}^{2}} \cdot \mathbf{Y}_{v, n}^{m}\left(\theta_{2}, \phi_{2}\right) \\
& =(2 n+1)\left[\frac{d^{2} P_{n+\nu}(\cos \Theta)}{d \Theta^{2}} \cos ^{2} \chi_{1}+\frac{d P_{n+v}(\cos \Theta)}{d \Theta} \sin ^{2} \chi_{1} \cot \Theta\right], \\
& \sum_{m=-n}^{n} \frac{\partial \overline{\mathbf{Y}_{v, n}^{m}\left(\theta_{1}, \phi_{1}\right)}}{\partial \theta_{1}} \cdot \frac{\partial \mathbf{Y}_{v, n}^{m}\left(\theta_{2}, \phi_{2}\right)}{\partial \theta_{2}} \\
& =(2 n+1)\left[\frac{d^{2} P_{n+\nu}(\cos \Theta)}{d \Theta^{2}} \cos \chi_{1} \cos \chi_{2}-\frac{d P_{n+\nu}(\cos \Theta)}{d \Theta} \frac{\sin \chi_{1} \sin \chi_{2}}{\sin \Theta}\right],
\end{aligned}
$$

$$
\begin{aligned}
& \sum_{m=-n}^{n} \frac{\partial \overline{\mathbf{Y}_{v, n}^{m}\left(\theta_{1}, \phi_{1}\right)}}{\partial \theta_{1}} \cdot \frac{1}{\sin \theta_{2}} \frac{\partial \mathbf{Y}_{v, n}^{m}\left(\theta_{2}, \phi_{2}\right)}{\partial \phi_{2}} \\
& =-(2 n+1)\left[\frac{d^{2} P_{n+v}(\cos \Theta)}{d \Theta^{2}} \cos \chi_{1} \sin \chi_{2}+\frac{d P_{n+v}(\cos \Theta)}{d \Theta} \frac{\sin \chi_{1} \cos \chi_{2}}{\sin \Theta}\right], \\
& \sum_{m=-n}^{n} \frac{\partial}{\partial \theta_{1}}\left[\frac{1}{\sin \theta_{1}} \frac{\partial \overline{\mathbf{Y}_{v, n}^{m}\left(\theta_{1}, \phi_{1}\right)}}{\partial \phi_{1}}\right] \cdot \mathbf{Y}_{v, n}^{m}\left(\theta_{2}, \phi_{2}\right)
\end{aligned}
$$$$
=(2 n+1)\left[\frac{d^{2} P_{n+\nu}(\cos \Theta)}{d \Theta^{2}}-\frac{d P_{n+\nu}(\cos \Theta)}{d \Theta} \cot \Theta\right] \cos \chi_{1} \sin \chi_{1},
$$$$
\sum_{m=-n}^{n} \frac{1}{\sin \theta_{1}} \frac{\partial}{\partial \phi_{1}}\left[\frac{\partial \overline{\mathbf{Y}_{v, n}^{m}\left(\theta_{1}, \phi_{1}\right)}}{\partial \theta_{1}}\right] \cdot \mathbf{Y}_{v, n}^{m}\left(\theta_{2}, \phi_{2}\right)
$$

$$
\begin{aligned}
& =(2 n+1)\left[\frac{d^{2} P_{n+\nu}(\cos \Theta)}{d \Theta^{2}} \cos \chi_{1} \sin \chi_{1}+\frac{d P_{n+\nu}(\cos \Theta)}{d \Theta} \frac{\sin ^{2} \chi_{1} \cot \chi_{2}}{\sin \Theta}\right], \\
& \sum_{m=-n}^{n} \frac{1}{\sin \theta_{1}} \frac{\partial \overline{\mathbf{Y}_{v, n}^{m}\left(\theta_{1}, \phi_{1}\right)}}{\partial \phi_{1}} \cdot \frac{1}{\sin \theta_{2}} \frac{\partial \mathbf{Y}_{v, n}^{m}\left(\theta_{2}, \phi_{2}\right)}{\partial \phi_{2}}
\end{aligned}
$$$$
=(2 n+1)\left[-\frac{d^{2} P_{n+v}(\cos \Theta)}{d \theta^{2}} \sin \chi_{1} \sin \chi_{2}+\frac{d P_{n+v}(\cos \Theta)}{d \Theta} \frac{\cos \chi_{1} \cos \chi_{2}}{\sin \Theta}\right] \text {, }
$$

$$
\sum_{m=-n}^{n} \frac{1}{\sin ^{2} \theta_{1}} \frac{\partial^{2} \overline{\mathbf{Y}_{v, n}\left(\theta_{1}, \phi_{1}\right)}}{\partial \phi_{1}^{2}} \cdot \mathbf{Y}_{n}^{m}\left(\theta_{2}, \phi_{2}\right)
$$$$
=(2 n+1)\left[\frac{d^{2} P_{n}^{m}(\cos \Theta)}{d \Theta^{2}} \sin ^{2} \chi_{1}-\frac{d P_{n}(\cos \Theta)}{d \Theta} \frac{\sin \chi_{1} \cos \chi_{1}}{\sin \Theta} \cot \chi_{2}\right] .
$$

These lead to the following special cases when $\Theta=0$, and $\theta_{1}=\theta_{2}, \phi_{1}=\phi_{2}, \chi_{1}=\pi$, $\chi_{2}=0$,

$$
\sum_{m=-n}^{n}\left|\mathbf{Y}_{v, n}^{m}(\theta, \phi)\right|^{2}=(2 n+1)
$$




$$
\begin{aligned}
\sum_{m=-n}^{n} \frac{\partial \overline{\mathbf{Y}_{v, n}^{m}(\theta, \phi)}}{\partial \theta} \cdot \mathbf{Y}_{v, n}^{m}(\theta, \phi) & =0 \\
\sum_{m=-n}^{n} \frac{1}{\sin \theta} \frac{\partial \overline{\mathbf{Y}_{v, n}^{m}(\theta, \phi)}}{\partial \phi} \cdot \mathbf{Y}_{v, n}^{m}(\theta, \phi) & =0 \\
\sum_{m=-n}^{n} \frac{\partial^{2} \overline{\mathbf{Y}_{v, n}^{m}(\theta, \phi)}}{\partial \theta^{2}} \cdot \mathbf{Y}_{v, n}^{m}(\theta, \phi) & =-\frac{1}{2}(2 n+1)(n+v)(n+v+1) \\
\left.\sum_{m=-n}^{n} \frac{\partial \overline{\mathbf{Y}_{v, n}^{m}(\theta, \phi)}}{\partial \theta} \cdot \frac{1}{\sin \theta} \frac{\partial \mathbf{Y}_{v, n}^{m}(\theta, \phi)}{\partial \theta}\right|^{2} & =\frac{1}{2}(2 n+1)(n+v)(n+v+1) \\
\sum_{m=-n}^{n}\left|\frac{1}{\sin \theta} \frac{\partial \mathbf{Y}_{v, n}^{m}(\theta, \phi)}{\partial \phi}\right|^{2} & =\frac{1}{2}(2 n+1)(n+v)(n+v+1)
\end{aligned}
$$

The tensor form of the addition theorem given in (73) can also be differentiated to yield formulae analogous to those of (74) to (81). Perhaps we need record here only three of the special cases

$$
\begin{gathered}
\sum_{\zeta=-1}^{1} \sum_{m=-n}^{n} \frac{\overline{\mathbf{Y}_{\zeta, n+\nu}^{m}(\theta, \phi)} \mathbf{Y}_{\zeta, n+v}^{m}(\theta, \phi)=(2 n+2 \nu+1) \mathrm{U},}{\sum_{\zeta=-1}^{1} \sum_{m=-n}^{n} \frac{\partial \overline{\mathbf{Y}_{\zeta, n+v}^{m}(\theta, \phi)}}{\partial \theta} \frac{\partial \mathbf{Y}_{\zeta, n+\nu}^{m}(\theta, \phi)}{\partial \theta}} \\
=\frac{1}{2}(2 n+2 v+1)(n+v)(n+v+1) \mathrm{U} \\
\sum_{\zeta=-1}^{1} \sum_{m=-n}^{n} \frac{1}{\sin ^{2} \theta} \frac{\partial \overline{\mathbf{Y}_{\zeta, n+v}^{m}(\theta, \phi)}}{\partial \phi} \frac{\partial \mathbf{Y}_{\zeta, n+v}^{m}(\theta, \phi)}{\partial \phi} \\
=\frac{1}{2}(2 n+2 v+1)(n+\nu)(n+v+1) \mathrm{U}
\end{gathered}
$$

\section{Tensor spherical harmonics of the second rank}

Tensor spherical harmonics can be defined in a number of ways, but the systematic method of James [5], which allows repeated generation up to any rank, will be used here, namely

$$
\mathrm{Y}_{\zeta, \nu, n}^{m}(\theta, \phi)=(-1)^{n-m} \sqrt{2 n+1} \sum_{\mu=-1}^{1}\left(\begin{array}{ccc}
n & n+\nu & 1 \\
m & -m+\mu & -\mu
\end{array}\right) \mathbf{Y}_{\zeta, n+\nu}^{m-\mu}(\theta, \phi) \mathbf{e}_{\mu},
$$

which corresponds to the following forms, as in (70) 


$$
\begin{aligned}
Y_{\zeta, 1, n}^{m}(\theta, \phi)= & \frac{1}{\sqrt{(n+1)(2 n+3)}}\left[\sqrt{(n+m+1)(n+m+2) / 2} \mathbf{Y}_{\zeta, n+1}^{m+1}(\theta, \phi) \mathbf{e}_{-1}\right. \\
& -\sqrt{(n-m+1)(n+m+1)} \mathbf{Y}_{\zeta, n+1}^{m}(\theta, \phi) \mathbf{e}_{0} \\
& \left.+\sqrt{(n-m+1)(n-m+2) / 2} \mathbf{Y}_{\zeta, n+1}^{m-1}(\theta, \phi) \mathbf{e}_{1}\right] \\
Y_{\zeta, 0, n}^{m}(\theta, \phi)= & \frac{1}{\sqrt{n(n+1)}}\left[\sqrt{(n+m+1)(n-m) / 2} \mathbf{Y}_{\zeta, n}^{m+1}(\theta, \phi) \mathbf{e}_{-1}\right. \\
& +m \mathbf{Y}_{\zeta, n}^{m}(\theta, \phi) \mathbf{e}_{0} \\
& \left.-\sqrt{(n+m)(n-m+1) / 2} \mathbf{Y}_{\zeta, n}^{m-1}(\theta, \phi) \mathbf{e}_{1}\right] \\
Y_{\zeta,-1, n}^{m}(\theta, \phi)= & \frac{1}{\sqrt{n(2 n-1)}}\left[\sqrt{(n-m-1)(n-m) / 2} \mathbf{Y}_{\zeta, n-1}^{m+1}(\theta, \phi) \mathbf{e}_{-1}\right. \\
& +\sqrt{(n+m)(n-m)} \mathbf{Y}_{\zeta, n-1}^{m}(\theta, \phi) \mathbf{e}_{0} \\
& \left.+\sqrt{(n+m+1)(n+m) / 2} \mathbf{Y}_{\zeta, n-1}^{m-1}(\theta, \phi) \mathbf{e}_{1}\right]
\end{aligned}
$$

In operator form, the nine tensor spherical harmonics are given by

$$
\begin{aligned}
\sqrt{(n+2)(2 n+3)} \mathrm{Y}_{1,1, n}^{m}(\theta, \phi) & =r^{n+3} \nabla\left[\frac{1}{r^{n+2}} \mathbf{Y}_{1, n}^{m}(\theta, \phi)\right], \\
\sqrt{(n+1)(n+2)} \mathrm{Y}_{0,1, n}^{m}(\theta, \phi) & =\mathbf{L} \mathbf{Y}_{1, n}^{m}(\theta, \phi), \\
\sqrt{(n+1)(2 n+3)} \mathrm{Y}_{-1,1, n}^{m}(\theta, \phi) & =\frac{1}{r^{n}} \nabla\left[r^{n+1} \mathbf{Y}_{1, n}^{m}(\theta, \phi)\right], \\
\sqrt{(n+1)(2 n+1)} \mathrm{Y}_{1,0, n}^{m}(\theta, \phi) & =r^{n+2} \nabla\left[\frac{1}{r^{n+1}} \mathbf{Y}_{0, n}^{m}(\theta, \phi)\right], \\
\sqrt{n(n+1)} \mathrm{Y}_{0,0, n}^{m}(\theta, \phi) & =\mathbf{L} \mathbf{Y}_{0, n}^{m}(\theta, \phi), \\
\sqrt{n(2 n+1)} \mathrm{Y}_{-1,0, n}^{m}(\theta, \phi) & =\frac{1}{r^{n-1}} \nabla\left[r^{n} \mathbf{Y}_{0, n}^{m}(\theta, \phi)\right], \\
\sqrt{n(2 n-1)} \mathrm{Y}_{1,-1, n}(\theta, \phi) & =r^{n+1} \nabla\left[\frac{1}{r^{n}} \mathbf{Y}_{-1, n}^{m}(\theta, \phi)\right], \\
\sqrt{(n-1) n} \mathrm{Y}_{0,-1, n}^{m}(\theta, \phi) & =\mathbf{L} \mathbf{Y}_{-1, n}^{m}(\theta, \phi), \\
\sqrt{(n-1)(2 n-1)} \mathrm{Y}_{-1,-1, n}^{m}(\theta, \phi) & =\frac{1}{r^{n-2}} \nabla\left[r^{n-1} \mathbf{Y}_{-1, n}^{m}(\theta, \phi)\right] .
\end{aligned}
$$

The scalar form of the addition theorem for tensor spherical harmonics is

$$
\sum_{m=-n}^{n} \overline{Y_{\zeta, v, n}^{m}\left(\theta_{1}, \phi_{1}\right)}: Y_{\zeta, v, n}^{m}\left(\theta_{2}, \phi_{2}\right)^{\top}=(2 n+1) P_{n+\zeta}(\cos \Theta)
$$


where the superscript $T$ denotes transpose. This addition theorem can be differentiated as before to yield a sequence of addition theorems. We record here only three special cases or sum rules

$$
\begin{aligned}
\sum_{m=-n}^{n} \overline{Y_{\zeta, v, n}^{m}(\theta, \phi)}: Y_{\zeta, v, n}^{m}(\theta, \phi)^{\top} & =(2 n+1), \\
\sum_{m=-n}^{n} \frac{\partial \overline{Y_{\zeta, v, n}^{m}(\theta, \phi)}}{\partial \theta}: \frac{\partial Y_{\zeta, v, n}^{m}(\theta, \phi)^{\top}}{\partial \theta} & =\frac{1}{2}(2 n+1)(n+v)(n+v+1), \\
\sum_{m=-n}^{n} \frac{1}{\sin ^{2} \theta} \frac{\partial \overline{Y_{\zeta, v, n}^{m}(\theta, \phi)}}{\partial \phi} & : \frac{\partial Y_{\zeta, v, n}^{m}(\theta, \phi)^{\top}}{\partial \phi} \\
& =\frac{1}{2}(2 n+1)(n+v)(n+v+1) .
\end{aligned}
$$

The three equations (97)-(99) each contain nine separate special cases or sum rules. Using the spherical polar forms for the tensors given by James [5], a typical such special case obtained, for example, from (97) with $\zeta=1, v=1$, is

$$
\begin{gathered}
\sum_{m=-n}^{n}\left\{(n+1)^{2}(n+2)^{2}\left|Y_{n}^{m}(\theta, \phi)\right|^{2}+2(n+2)^{2}\left|\frac{\partial Y_{n}^{m}(\theta, \phi)}{\partial \theta}\right|^{2}+\right. \\
+2(n+2)^{2}\left|\frac{1}{\sin \theta} \frac{\partial Y_{n}^{m}(\theta, \phi)}{\partial \phi}\right|^{2}+\left|\frac{\partial^{2} Y_{n}^{m}(\theta, \phi)}{\partial \theta^{2}}-(n+1) Y_{n}^{m}(\theta, \phi)\right|^{2}+ \\
\left.+\left|\frac{\partial^{2} Y_{n}^{m}(\theta, \phi)}{\partial \theta^{2}}+(n+1)^{2} Y_{n}^{m}(\theta, \phi)\right|^{2}+2\left|\frac{\partial}{\partial \theta}\left[\frac{1}{\sin \theta} \frac{\partial Y_{n}^{m}(\theta, \phi)}{\partial \phi}\right]\right|^{2}\right\} \\
=(n+1)(n+2)(2 n+1)(2 n+3),
\end{gathered}
$$

which can be verified using the sum rules given in Section 4 .

\section{Addition theorems for Chebyshev functions}

The Chebyshev polynomials of the second kind, $u_{p}(\cos \psi)$, are defined by

$$
u_{p}(\cos \psi)=\frac{\sin (p+1) \psi}{(p+1) \sin \psi}
$$

and the first few are given by

$$
\begin{aligned}
& u_{0}(\cos \psi)=1, \quad u_{1}(\cos \psi)=\cos \psi, \\
& u_{2}(\cos \psi)=\frac{4}{3} \cos ^{2} \psi-\frac{1}{3}
\end{aligned}
$$




$$
\begin{aligned}
& u_{3}(\cos \psi)=2 \cos ^{3} \psi-\cos \psi, \\
& u_{4}(\cos \psi)=\frac{16}{5} \cos ^{4} \psi-\frac{12}{5} \cos ^{2} \psi+\frac{1}{5}, \\
& u_{5}(\cos \psi)=\frac{16}{3} \cos ^{5} \psi-\frac{16}{3} \cos ^{3} \psi+\cos \psi, \\
& u_{6}(\cos \psi)=\frac{64}{7} \cos ^{6} \psi-\frac{80}{7} \cos ^{4} \psi+\frac{24}{7} \cos ^{2} \psi-\frac{1}{7} .
\end{aligned}
$$

The associated Chebyshev functions of the second kind, $u_{p}^{n}(\cos \psi)$, are defined by

$$
u_{p}^{n}(\cos \psi)=\left[(2 n+1)(p+1) \frac{(p-n) !}{(p+n+1) !}\right]^{1 / 2} \sin ^{n} \psi\left(\frac{d}{d \cos \psi}\right)^{n} u_{p}(\cos \psi),
$$

so that $u_{p}^{0}(\cos \psi)=u_{p}(\cos \psi)$. The first few are found to be

$$
\begin{aligned}
& u_{1}^{1}(\cos \psi)=\sin \psi, \\
& u_{2}^{1}(\cos \psi)=\frac{2 \sqrt{6}}{3} \cos \psi \sin \psi, \\
& u_{2}^{2}(\cos \psi)=\frac{2 \sqrt{2}}{3} \sin ^{2} \psi, \\
& u_{3}^{1}(\cos \psi)=\frac{\sqrt{5}}{5}\left(6 \cos ^{2} \psi-1\right) \sin \psi, \\
& u_{3}^{2}(\cos \psi)=2 \cos \psi \sin ^{2} \psi, \\
& u_{3}^{3}(\cos \psi)=\frac{2 \sqrt{5}}{5} \sin ^{3} \psi, \\
& u_{4}^{1}(\cos \psi)=\frac{2 \sqrt{2}}{5}\left(8 \cos ^{2} \psi-3\right) \cos \psi \sin \psi, \\
& u_{4}^{2}(\cos \psi)=\frac{2 \sqrt{75}}{35}\left(8 \cos ^{2} \psi-1\right) \sin ^{2} \psi, \\
& u_{4}^{3}(\cos \psi)=\frac{8 \sqrt{2}}{5} \cos \psi \sin ^{3} \psi, \\
& u_{4}^{4}(\cos \psi)=\frac{8 \sqrt{14}}{35} \sin ^{4} \psi .
\end{aligned}
$$

The associated Chebyshev functions arise as solutions of the Laplace equation in four dimensions, and with the parameterization of the four dimensional Cartesian space $(x, y, z, t)$ by means of

$$
\begin{aligned}
& x=r \sin \psi \sin \theta \cos \phi, \\
& y=r \sin \psi \sin \theta \sin \phi, \\
& z=r \sin \psi \cos \theta, \\
& t=r \cos \psi,
\end{aligned}
$$

the separable solutions of the Laplace equation, $\nabla^{2} V=0$, are found to be of the form

$$
\begin{aligned}
V=\sum_{p=0}^{\infty} \sum_{n=0}^{p} \sum_{m=0}^{n} & \left(A_{p n}^{m} r^{p}+B_{p n}^{m} r^{-p-2}\right) \times \\
& \times\left(C_{p n}^{m} \cos m \phi+D_{p n}^{n} \sin m \phi\right) P_{n}^{m}(\cos \theta) u_{p}^{n}(\cos \psi),
\end{aligned}
$$


the individual functions forming an orthogonal set under integration over the unit $(r=1)$ three-dimensional hypersphere parameterized by the angular coordinates $(\psi, \theta, \phi)$ and embedded in the four-dimensional space $(x, y, z, t)$.

The basic addition theorem for the Chebyshev polynomials is a special case of a general addition theorem for Gegenbauer polynomials first given by Gegenbauer [4] and is

$$
\sum_{n=0}^{p} u_{p}^{n}\left(\cos \psi_{1}\right) u_{p}^{n}\left(\cos \psi_{2}\right) P_{n}(\cos \Theta)=u_{p}(\cos \Psi),
$$

which in its simplest case $p=1$ defines the angle $\Psi$ by

$$
\cos \Psi=\cos \psi_{1} \cos \psi_{2}+\sin \psi_{1} \sin \psi_{2} \cos \Theta
$$

A rotation through an angle $2 \psi$, about an axis with polar coordinates $(\theta, \phi)$, can be specified in terms of a quaternion with components $\lambda, \mu, \nu, \rho$ defined by

$\lambda=\sin \psi \sin \theta \cos \phi, \quad \mu=\sin \psi \sin \theta \sin \phi, \quad \nu=\sin \psi \cos \theta, \quad \rho=\cos \psi$.

On forming the complex Cayley-Klein parameters $u$ and $v$, where

$$
u=\rho-i v, \quad v=-\mu-i \lambda,
$$

the result of two successive rotations is given by the $S U(2)$ product

$$
\left(\begin{array}{cc}
u_{3} & v_{3} \\
-\bar{v}_{3} & \bar{u}_{3}
\end{array}\right)=\left(\begin{array}{cc}
u_{2} & v_{2} \\
-\bar{v}_{2} & \bar{u}_{2}
\end{array}\right)\left(\begin{array}{cc}
u_{1} & v_{1} \\
-\bar{v}_{1} & \bar{u}_{1}
\end{array}\right)
$$

and this implies the following two relations and their conjugates,

$$
u_{3}=u_{2} u_{1}-v_{2} \bar{v}_{1}, \quad v_{3}=u_{2} v_{1}+v_{2} \bar{u}_{1} \text {. }
$$

An overbar is used to denote complex conjugate. The results of (110) can be written in matrix form as

$$
\left(\begin{array}{l}
\lambda_{3} \\
\mu_{3} \\
\nu_{3} \\
\rho_{3}
\end{array}\right)=\left(\begin{array}{cccc}
\rho_{2} & -\nu_{2} & \mu_{2} & \lambda_{2} \\
\nu_{2} & \rho_{2} & -\lambda_{2} & \mu_{2} \\
-\mu_{2} & \lambda_{2} & \rho_{2} & \nu_{2} \\
-\lambda_{2} & -\mu_{2} & -\nu_{2} & \rho_{2}
\end{array}\right)\left(\begin{array}{l}
\lambda_{1} \\
\mu_{1} \\
\nu_{1} \\
\rho_{1}
\end{array}\right) .
$$

The equation for $\rho_{3}$ is now given by

$$
\begin{aligned}
\cos \psi_{3} & =\cos \psi_{1} \cos \psi_{2}-\sin \psi_{1} \sin \psi_{2}\left[\cos \theta_{1} \cos \theta_{2}+\sin \theta_{1} \sin \theta_{2} \cos \left(\phi_{1}-\phi_{2}\right)\right] \\
& =\cos \psi_{1} \cos \psi_{2}+\sin \psi_{1} \sin \psi_{2} \cos (\pi-\Theta)
\end{aligned}
$$


showing that to reconcile the basic result of the addition theorem and that of the quaternion formula for the combination of successive rotations, the resultant rotation angle $2 \psi_{3}$ from the quaternion formula can be equated with the angle $2 \Psi$ of the addition formula, only if $\Theta$ of the addition theorem is replaced by its complement $\pi-\Theta$.

By virtue of (108), a rotation can be thought of as being specified by a point on the unit three-dimensional hypersphere in a four-dimensional space, and that distributions of rotations can be specified in terms of the functions given in (106) with $r=1$, for example, Roberts and Winch [6].

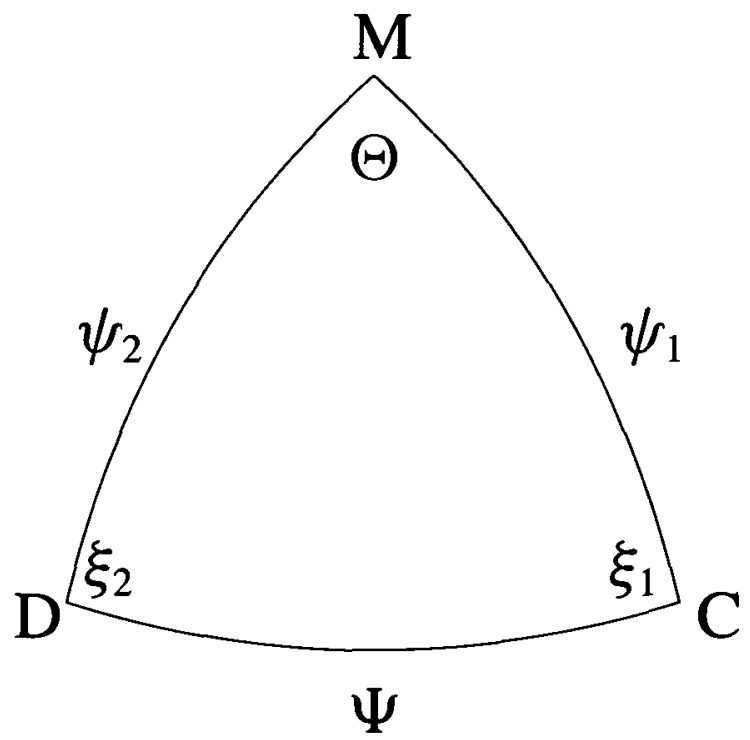

FIGURE 2. Nomenclature used on the hypersphere. Note that $\Theta$ of Figure 1 becomes an internal angle of the spherical triangle.

Let $C\left(\psi_{1}, \theta_{1}, \phi_{1}\right)$ and $D\left(\psi_{2}, \theta_{2}, \phi_{2}\right)$ be points on the unit hypersphere, corresponding to rotations through angles $2 \psi_{1}$ and $2 \psi_{2}$, about axes with polar coordinates $\left(\theta_{1}, \phi_{1}\right)$, $\left(\theta_{2}, \phi_{2}\right)$, respectively. Then the cosine rule

$$
\cos \Theta=\cos \theta_{1} \cos \theta_{2}+\sin \theta_{1} \sin \theta_{2} \cos \left(\phi_{1}-\phi_{2}\right),
$$

gives the great circle distance $\Theta$ between the rotation axes. In the following we shall use, from the general addition theorem

$$
\cos \Psi=\cos \psi_{1} \cos \psi_{2}+\sin \psi_{1} \sin \psi_{2} \cos \Theta,
$$


which is the scalar product of two unit vectors corresponding to $\left(\lambda_{1}, \mu_{1}, \nu_{1}, \rho_{1}\right)$ and $\left(\lambda_{2}, \mu_{2}, \nu_{2}, \rho_{2}\right)$ and is therefore between -1 and 1 , so that $\Psi$ is a real angle.

The determination of the various addition theorems requires the derivatives of the angles $\Psi, \chi_{1}$ and $\chi_{2}$ with respect to $\psi_{1}, \psi_{2}$ and $\Theta$ and these follow directly from the results of Section 2, with substitutions which are obvious from a comparison of Figures 1 and 2:

$$
\begin{array}{lll}
\frac{\partial \Psi}{\partial \psi_{1}}=\cos \xi_{1}, & \frac{\partial \Psi}{\partial \psi_{2}}=\cos \xi_{2}, \\
\frac{\partial \Psi}{\partial \Theta}=\sin \psi_{1} \sin \xi_{1}, & \frac{\partial \Psi}{\partial \Theta}=\sin \psi_{2} \sin \xi_{2}, \\
\frac{\partial \xi_{1}}{\partial \psi_{1}}=-\sin \xi_{1} \cot \Psi, & \frac{\partial \xi_{1}}{\partial \psi_{2}}=\frac{\sin \xi_{2}}{\sin \Psi}, \\
\frac{\partial \xi_{1}}{\partial \Theta}=-\frac{\sin \psi_{1} \sin \xi_{1}}{\sin \Psi} \cot \psi_{2}, & \frac{\partial \xi_{1}}{\partial \Theta}=-\frac{\sin \psi_{2} \cos \xi_{2}}{\sin \Psi}, \\
\frac{\partial \xi_{2}}{\partial \psi_{1}}=\frac{\sin \xi_{1}}{\sin \Psi} & \frac{\partial \xi_{2}}{\partial \psi_{2}}=-\sin \xi_{2} \cot \Psi, \\
\frac{\partial \xi_{2}}{\partial \Theta}=-\frac{\sin \psi_{1} \cos \xi_{1}}{\sin \Psi}, & \frac{\partial \xi_{2}}{\partial \Theta}=-\frac{\sin \psi_{2} \sin \xi_{2}}{\sin \Psi} \cot \xi_{1} .
\end{array}
$$

Differentiation of the addition theorem, (107), with respect to $\psi_{1}$ and $\Theta$, gives directly that

$$
\begin{aligned}
\sum_{n=0}^{p} \frac{d u_{p}^{n}\left(\cos \psi_{1}\right)}{d \psi_{1}} u_{p}^{n}\left(\cos \psi_{2}\right) P_{n}(\cos \Theta) & =\frac{d u_{p}(\cos \Psi)}{d \Psi} \cos \xi_{1}, \\
\sum_{n=0}^{p} \frac{1}{\sin \psi_{1}} u_{p}^{n}\left(\cos \psi_{1}\right) u_{p}^{n}\left(\cos \psi_{2}\right) \frac{d P_{n}(\cos \Theta)}{d \Theta} & =\frac{d u_{p}(\cos \Psi)}{d \Psi} \sin \xi_{1} .
\end{aligned}
$$

Differentiating (114) with respect to $\psi_{1}, \psi_{2}$ and $\Theta$, gives the results

$$
\begin{aligned}
& \sum_{n=0}^{p} \frac{d^{2} u_{p}^{n}\left(\cos \psi_{1}\right)}{d \psi_{1}^{2}} u_{p}^{n}\left(\cos \psi_{2}\right) P_{n}(\cos \Theta) \\
& \quad=\frac{d^{2} u_{p}(\cos \Psi)}{d \Psi^{2}} \cos ^{2} \xi_{1}+\frac{d u_{p}(\cos \Psi)}{d \Psi} \sin ^{2} \xi_{1} \cot \Psi \\
& \sum_{n=0}^{p} \frac{d u_{p}^{n}\left(\cos \psi_{1}\right)}{d \psi_{1}} \frac{d u_{p}^{n}\left(\cos \psi_{2}\right)}{d \psi_{2}} P_{n}(\cos \Theta) \\
& \quad=\frac{d^{2} u_{p}(\cos \Psi)}{d \Psi^{2}} \cos \xi_{1} \cos \xi_{2}-\frac{d u_{p}(\cos \Psi)}{d \Psi} \frac{\sin \xi_{1} \sin \xi_{2}}{\sin \Psi} \\
& \sum_{n=0}^{p} \frac{1}{\sin \psi_{1}} \frac{d u_{p}^{n}\left(\cos \psi_{1}\right)}{d \psi_{1}} u_{p}^{n}\left(\cos \psi_{2}\right) \frac{d P_{n}(\cos \Theta)}{d \Theta} \\
& =\frac{d^{2} u_{p}(\cos \Psi)}{d \Psi^{2}} \cos \xi_{1} \sin \xi_{1}+\frac{d u_{p}(\cos \Psi)}{d \Psi} \frac{\sin ^{2} \xi_{1} \cot \xi_{2}}{\sin \Psi} .
\end{aligned}
$$


Differentiate (119) with respect to $\psi_{1}$ and $\Theta$ to obtain

$$
\begin{aligned}
& \sum_{n=0}^{p} \frac{d}{d \psi_{1}} \quad\left[\frac{1}{\sin \psi_{1}} u_{p}^{n}\left(\cos \psi_{1}\right)\right] u_{p}^{n}\left(\cos \psi_{2}\right) \frac{d P_{n}(\cos \Theta)}{d \Theta} \\
& =\left[\frac{d^{2} u_{p}(\cos \Psi)}{d \Psi^{2}}-\frac{d u_{p}(\cos \Psi)}{d \Psi} \cot \Psi\right] \sin \xi_{1} \cos \xi_{1}, \\
& \sum_{n=0}^{p} \frac{1}{\sin \psi_{1}} u_{p}^{n}\left(\cos \psi_{1}\right) \frac{1}{\sin \psi_{2}} u_{p}^{n}\left(\cos \psi_{2}\right) \frac{d^{2} P_{n}(\cos \Theta)}{d \Theta^{2}} \\
& =\frac{d^{2} u_{p}(\cos \Psi)}{d \Psi^{2}} \sin \xi_{1} \sin \xi_{2}-\frac{d u_{p}(\cos \Psi)}{d \Psi} \frac{\cos \xi_{1} \cos \xi_{2}}{\sin \Psi} .
\end{aligned}
$$

In the special case

$$
\Psi=\psi_{1}-\psi_{2}, \quad \xi_{1}=0, \quad \xi_{2}=\pi, \quad \Theta=0,
$$

we obtain the following duplication formulae from (113), (114), (116), (117) and (120), respectively

$$
\begin{aligned}
\sum_{n=0}^{p} u_{p}^{n}\left(\cos \psi_{1}\right) u_{p}^{n}\left(\cos \psi_{2}\right) & =u_{p}\left[\cos \left(\psi_{1}-\psi_{2}\right)\right] \\
\sum_{n=0}^{p} \frac{d u_{p}^{n}\left(\cos \psi_{1}\right)}{d \psi_{1}} u_{p}^{n}\left(\cos \psi_{2}\right) & =\left[\frac{d u_{p}(\cos \Psi)}{d \Psi}\right]_{\Psi=\psi_{1}-\psi_{2}}, \\
\sum_{n=0}^{p} \frac{d^{2} u_{p}^{n}\left(\cos \psi_{1}\right)}{d \psi_{1}^{2}} u_{p}^{n}\left(\cos \psi_{2}\right) & =\left[\frac{d^{2} u_{p}(\cos \Psi)}{d \Psi^{2}}\right]_{\Psi=\psi_{1}-\psi_{2}}, \\
\sum_{n=0}^{p} \frac{d u_{p}^{n}\left(\cos \psi_{1}\right)}{d \psi_{1}} \frac{d u_{p}^{n}\left(\cos \psi_{2}\right)}{d \psi_{2}} & =-\left[\frac{d^{2} u_{p}(\cos \Psi)}{d \Psi^{2}}\right]_{\Psi=\psi_{1}-\psi_{2}} \\
\sum_{n=0}^{p} \frac{1}{2} n(n+1) \frac{1}{\sin \psi_{1}} u_{p}^{n}\left(\cos \psi_{1}\right) \frac{1}{\sin \psi_{2}} u_{p}^{n}\left(\cos \psi_{2}\right) & =-\left[\frac{1}{\sin \Psi} \frac{d u_{p}(\cos \Psi)}{d \Psi}\right]_{\Psi=\psi_{1}-\psi_{2}} .
\end{aligned}
$$

In the further special cases, making use of the results

$$
\begin{aligned}
{\left[\frac{1}{\sin \Psi} \frac{\left.d u_{p}(\cos \Psi)\right]_{\Psi=0}}{d \Psi}\right.} & =1, \\
{\left[\frac{d u_{p}(\cos \Psi)}{d \Psi}\right]_{\Psi=0} } & =-\frac{1}{3} p(p+2), \\
{\left[\frac{d^{2} u_{p}(\cos \Psi)}{d \Psi^{2}}\right]_{\Psi=0} } & =0, \\
{\left[\begin{array}{l}
2 \\
\end{array}\right.} & =-\frac{1}{3} p(p+2),
\end{aligned}
$$


the duplication formulae, (121)-(125), reduce to the following sums

$$
\begin{aligned}
\sum_{n=0}^{p}\left[u_{p}^{n}(\cos \psi)\right]^{2} & =1, \\
\sum_{n=0}^{p} \frac{d u_{p}^{n}(\cos \psi)}{d \psi} u_{p}^{n}(\cos \psi) & =0, \\
\sum_{n=0}^{p} \frac{d^{2} u_{p}^{n}(\cos \psi)}{d \psi^{2}} u_{p}^{n}(\cos \psi) & =-\frac{1}{3} p(p+2), \\
\sum_{n=0}^{p}\left[\frac{d u_{p}^{n}(\cos \psi)}{d \psi}\right]^{2} & =\frac{1}{3} p(p+2), \\
\sum_{n=0}^{p} \frac{1}{2} n(n+1)\left[\frac{u_{p}^{n}(\cos \psi)}{\sin \psi}\right]^{2} & =\frac{1}{3} p(p+2) .
\end{aligned}
$$

The derivatives required in (126)-(129) may be obtained by using the hypergeometric form for the Chebyshev polynomials,

$$
u_{p}(\mu)={ }_{2} F_{1}\left(-p, p+2 ; \frac{3}{2} ; \frac{1}{2}(1-\mu)\right) \text {, }
$$

where $\mu=\cos \psi$. The $r^{\text {th }}$ derivative evaluated at $\mu=1$ is therefore given by

$$
\left[\left(\frac{d}{d \mu}\right)^{r} u_{p}(\mu)\right]_{\mu=1}=\frac{1}{p+1} \frac{(p+2) !}{(p-1) !} \frac{2^{r}}{(2 r+1) !} .
$$

\section{Acknowledgement}

Part of this work was completed whilst one of us (DEW) was on Special Studies Program leave at the School of Physics, University of Newcastle upon Tyne, U.K.

\section{References}

[1] S. Chapman and J. Bartels, Geomagnetism, (Oxford University Press, 1940) 621.

[2] A. T. Doodson, "The harmonic development of the tide generating potential", Proc. R. Soc. Lond. A 100 (1922) 305-329.

[3] W. M. Elsasser, "A statistical analysis of the earth's internal magnetic field", Phys. Rev. 60 (1941) 876-883.

[4] L. Gegenbauer, “Das additions Theorem der Functionen $C_{n}^{v}(x)$ ”, Wiener Sitzungsberichte 102 (1893) 942-950.

[5] R. W. James, "New tensor spherical harmonics, for application to the partial differential equations of mathematical physics", Phil. Trans. R. Soc., Lond., A 281 (1976) 195-221.

[6] P. H. Roberts and D. E. Winch, "On random rotations", Adv. Appl. Prob. 16 (1984) 638-655.

[7] K. A. Whaler and D. Gubbins, "Spherical harmonic analysis of the geomagnetic field: an example of a linear inverse problem", Geophys. J. R. Astr. Soc. 65 (1981) 645-693. 